\section{A Rare Giant Cell Tumor of the Tendon Sheath of the Dorsal Hand in a Child}

Dong-Han Lee, So Young Lim

Department of Plastic Surgery, Samsung Medical Center, Sungkyunkwan University School of Medicine, Seoul, Korea

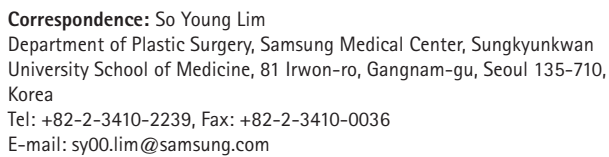

No potential conflict of interest relevant to this article was reported.

Received: 19 Jun 2014 • Revised: 25 Jun 2014 • Accepted: 25 Jun 2014 pISSN: 2234-6163 • elSSN: 2234-6171

http://dx.doi.org/10.5999/aps.2014.41.5.605 • Arch Plast Surg 2014;41:605-606 Copyright (C) 2014 The Korean Society of Plastic and Reconstructive Surgeon This is an Open Access article distributed under the terms of the Creative Commons Attribution Non-Commercial License (http://creativecommons.org/licenses/by-nc/3.0/) which permits unrestricted non-commercial use, distribution, and reproduction in any medium, provided the original work is properly cited.

Giant cell tumors of the tendon sheath (GCTTS) are benign soft tissue neoplasms of the synovial lining. The peak incidence occurs in the third to fifth decades of life with a slight female predominance, and is very rarely reported in children under the age of 10 years

[1]. GCTTS occurs as a firm, nodular mass, most commonly on the volar aspects of the fingers and hands [2]. In this article, we report a rare case of a child who developed a GCTTS in the dorsal aspect of the hand.

An 8-year-old boy presented to our clinic with a painless swelling on the dorsum of the right hand (Fig. 1). Medical history indicated that he had been healthy and had no underlying diseases. The mass was first noted one year prior to presentation, and had grown progressively since. At the first visit, the mass protruded from the metacarpal area of the right hand dorsum, and was approximately $2 \mathrm{~cm}$ in diameter (Fig. 1). The mass was firm, not freely movable, and was not tender. Magnetic resonance imaging (MRI) was performed to evaluate the

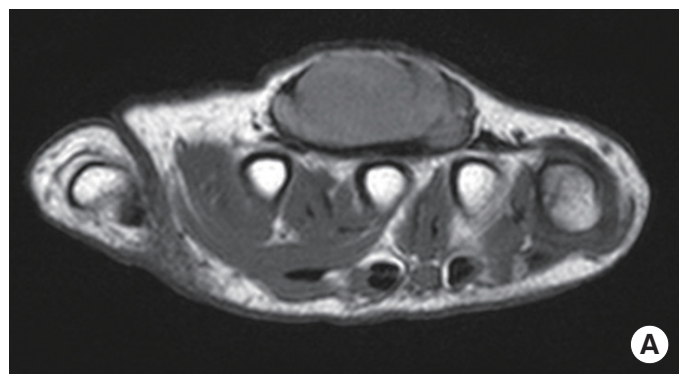

characteristics and boundaries of the mass. MRI revealed a $2.8 \times 3.3 \mathrm{~cm}$ lobulated subcutaneous soft tissue mass that was in contact with extensor digitorum tendon of the right hand, located dorsal to the second to fourth metacarpal bones (Fig. 2). It showed intermediate or dark signal intensity on both T1-weighted and T2-weighted images, and mild homogenous enhancement on contrast images. The mass was strongly suspected to be a giant cell tumor or fibroma originating from the tendon sheath.

Based on clinical findings and imaging, we decided to perform surgical excision of the mass with a suspicion of giant cell tumor or fibroma of the tendon sheath. The operation was performed under general anesthesia with surgical tourniquet. After making an incision in the middle of the swelling, we performed subcutaneous dissection. A poorly encapsulated mass was also successfully dissected and excised without sacrificing any tendons or neurovascular structures (Fig. 3). After irrigation and hemostasis, the incision was closed and a Penrose drain placed. The drain was removed on the second postoperative day, and the wound healed uneventfully. Cytological assays were negative for malignant cells and hemosiderin-laden macrophages, which would have implied previous hemorrhage. Pathologic findings revealed a $2.5 \times 1.5$ $\mathrm{cm}$ giant cell tumor of the tendon sheath (Fig. 4). The patient underwent an uneventful one-year course of postoperative follow-up without recurrence.

GCTTS is a slow-growing soft tissue mass that

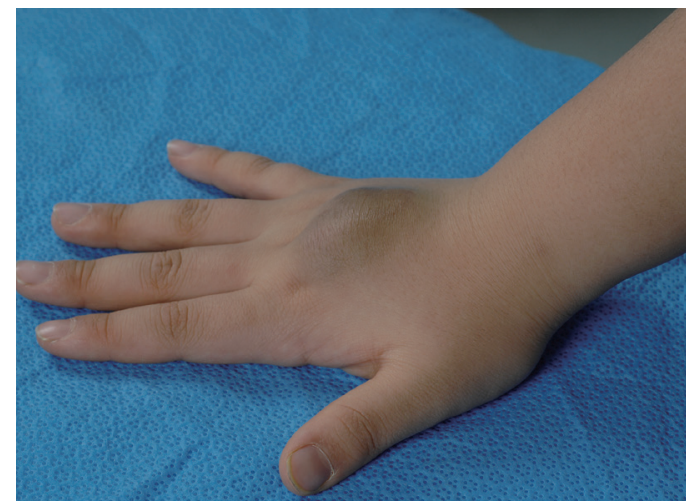

Fig. 1.

Preoperative clinical photograph of the patient's hand.

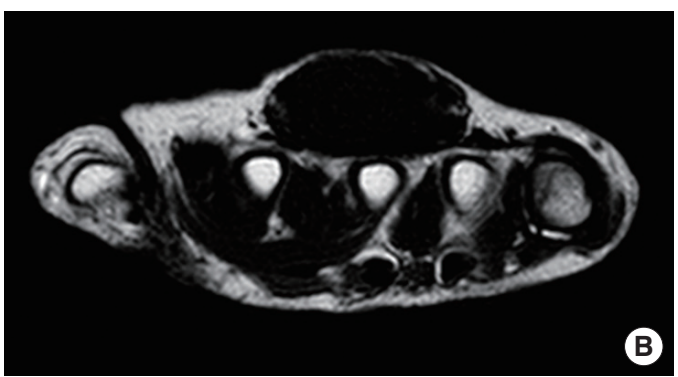

Fig. 2.

(A) A T1-weighted axial image shows a lobulated mass with intermediate signal intensity, adjacent to the extensor digitorum tendon. (B) A T2weighted axial image shows a mass with dark signal intensity. 


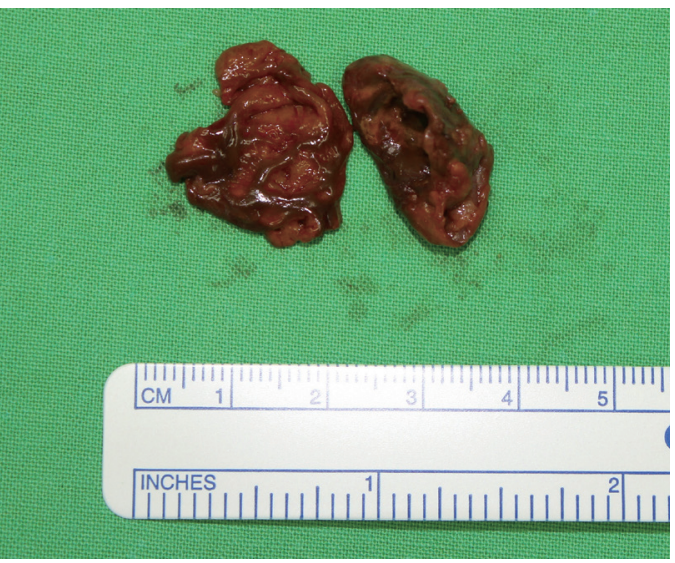

Fig. 3.

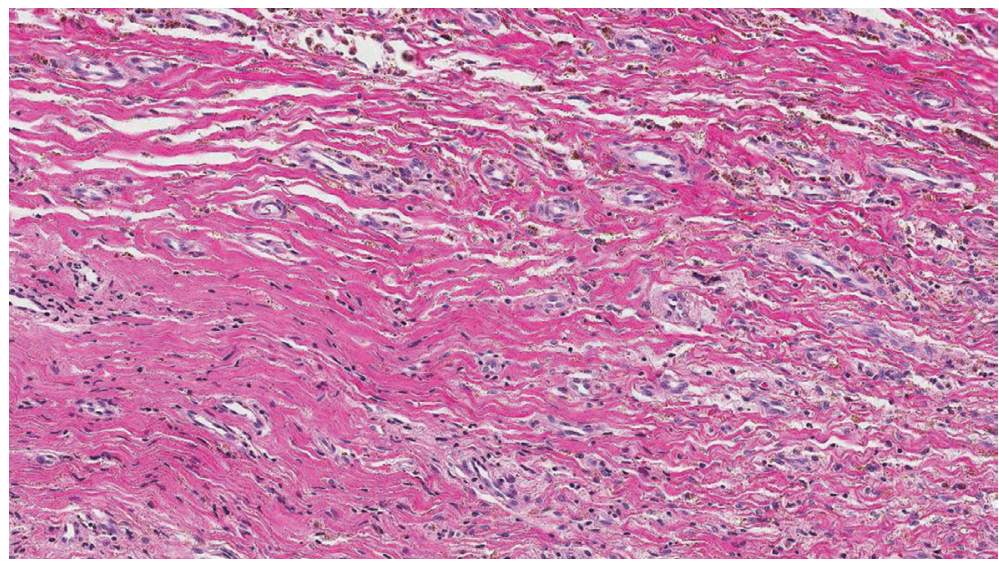

Fig. 4 .

Histology showing a giant cell tumor of the tendon sheath $\left(H \& E_{1} \times 200\right)$. poorly encapsulated mass.

develops over a period of months to years. The common clinical presentation is a painless, firm mass. Its radiological appearance is usually subtle, consisting only of soft tissue shadowing. Bony pressure erosions are reported to be more likely in recurrent cases [1]. GCTTS has many other names, including pigmented villonodular tenosynovitis, fibrous xanthoma, xanthogranuloma, and localized nodular synovitis, because its exact pathologic nature is unknown. Trauma, inflammation, metabolic disease, and neoplastic etiology are considered etiological factors [3]. It is the second most common tumor of the hand, but it is very uncommon in children under 10 years of age $[1,3]$.

Morphologically, GCTTS can be classified into a localized nodular type seen more commonly in the hand, and a diffuse type usually seen in larger joints [2]. Al-Qattan [4] classified GCTTS into two main types: Type I GCTTS is surrounded by one pseudocapsule, while type II is not surrounded by one pseudocapsule. The tumor in our case can be classified as type II GCTTS based upon the intraoperative findings. Histologically, GCTTS is characterized by a diverse cell population, including round stromal cells, multinucleated giant cells, and lipid-laden foam cells with deposits of hemosiderin [2].

GCTTS has high propensity for local recurrence, with reported recurrence rates varying widely from $4 \%$ to $44 \%$. The morphological factors associated with higher risk of recurrence include diffuse, poorly encapsulated lesions, presence of satellite lesions, involvement of tendon and joint, and intraosseous involvement [2]. There is consensus that recurrence is associated with incomplete excision. Thus, complete local excision remains the mainstay of GCTTS treatment $[3,5]$. All surrounding tissues should be examined for satellite lesions, and such lesions and connections to these lesions should be excised with the assistance of an operating microscope or a magnifying loupe. Local irradiation has been applied as an adjuvant therapy to prevent recurrence.

In the present study, we describe a rare case of GCTTS of the dorsal aspect of the hand in a child. As there is a high tendency for local recurrence, complete excision and prolonged follow-up is recommended when treating patients with GCTTS [1].

\section{References}

1. Hulse N, Watson SJ. Giant cell tumour of tendon sheath in a child: a case report. Hand Surg 2005; 10:97-100.

2. Lanzinger WD, Bindra R. Giant cell tumor of the tendon sheath. J Hand Surg Am 2013;38:154-7.

3. Suresh SS, Zaki H. Giant cell tumor of tendon sheath: case series and review of literature. J Hand Microsurg 2010;2:67-71.

4. Al-Qattan MM. Giant cell tumours of tendon sheath: classification and recurrence rate. J Hand Surg Br 2001; 26:72-5.

5. Adams EL, Yoder EM, Kasdan ML. Giant cell tumor of the tendon sheath: experience with 65 cases. Eplasty 2012;12:e50. 